\title{
Effect of Three Different Remineralizing Agents on Enamel Caries Formation - An in vitro Study
}

\section{Sathe $\mathrm{N},{ }^{1}$ Chakradhar Raju RVS, ${ }^{1}$ Chandrasekhar V ${ }^{2}$}

\author{
Department of Conservative Dentistry and \\ Endodontics \\ ${ }^{1}$ Sri Sai College of Dental Surgery \\ Andhra Pradesh, India \\ ${ }^{2}$ Mamata Dental College and Hospital \\ Andhra Pradesh, India
}

\section{Corresponding author}

Naresh Sathe

Department of Conservative Dentistry and Endodontics

Sri Sai College of Dental

Andhra Pradesh, India

Email: drnareshsathe@gmail.com

\section{Citation}

Sathe N, Chakradhar Raju RVS, Chandrasekhar V. Effect of 3 Different Remineralizing Agents on Enamel Caries Formation - An in-Vitro Study.

Kathmandu Univ Med J 2014;45(1):16-20.

\begin{abstract}
Background

Caries process is not a static one, but is dynamic with interspersed periods of demineralization and remineralization of enamel, intimately related and occurs episodically based upon the presence of cariogenic bacteria in dental plaque and the availability of refined carbohydrates for fermentation to organic acids.
\end{abstract}

\section{Objectives}

Early enamel caries could be reversed with avoidance of frank cavitation. The main objective of this study is to check wether enamel demineralization can be prevented by using the remineralizing agents.

\section{Method}

Forty freshly extracted human central incisors were selected and stored in saline at normal temperature. A window of $3 \times 3 \mathrm{~mm}$ enamel was created and all the specimens were then randomly divided into 4 groups of 10 each. Group I- teeth received no treatment, Group II- teeth treated with Acidulated Phosphate Flouride gel, Group III- teeth treated with Tooth Mousse Plus, Group IV- teeth treated with Remin+. Samples in all the groups were kept in artificial saliva for 24 hours and subjected to modified Ten Cate's solution at an acidic pH of 3.5 for 10 days. The samples were sectioned and subjected to SEM evaluation.

\section{Result}

Scanning Electron Microscope(SEM) images showed decrease in pore volume of the enamel in all the treatment groups compared to the control group indicating increase in resistance to demineralization in acidic $\mathrm{pH}$.

\section{Conclusion}

The three groups of remineralizing agents, Acidulated Phosphate Fluoride gel, Tooth Mousse Plus and Remin + showed significant increase in fluoride content and negligible increase in calcium content indicating there is remineraliztion.

\section{KEY WORDS}

Dental caries, hydroxyapatite, tooth demineralization, tooth remineralization

\section{INTRODUCTION}

Dental caries, gingivitis and periodontal disease are bacterial diseases that affect more people than any other disorder known to human kind. In children, dental caries is more common, attributing to their food habits. ${ }^{1}$ The caries process is not a static one, but is dynamic with interspersed periods of demineralization and remineralization. White spot lesions which were followed up for 6 to 7 years, either became arrested or reverted to sound enamel in $75 \%$ cases and only $25 \%$ cases progressed onto cavitation. ${ }^{2}$

The concept of preventing dental caries by vaccination has existed for almost as long as dental caries has been known to be an infectious disease process. The question of desirability of caries vaccine depends on economics and commercial considerations. ${ }^{3}$

The phenomenon that early enamel caries (white spot 
lesions) could be reversed with avoidance of frank cavitation was first recognized in the mid-1960. ${ }^{1}$ Both mineral loss and increase in lesion depth are markedly less with white spot lesions when compared with adjacent sound enamel. ${ }^{2}$ It was noted that reversion to sound enamel happened in about one third of white spot lesions, providing evidence that intra oral remineralization without progression to cavitation did occur.

With a limited time frame for remineralization of white spot lesions, several investigators worked toward developing calcifying fluids for reversal of intact enamel lesions. Unfortunately, calcifying fluids required prolonged and repeated exposures to arrest or remineralize white spot lesions. Innovative methods for reducing the length and number of exposures did provide a significant degree of remineralization in various in-vitro studies. In general, the dental practitioner has a limited armamentarium for combating dental caries progression. ${ }^{1}$

The physico-chemical properties of the mineral comprising the tooth surface and subsurface modulate the development, arrestment and remineralization of dental caries. Post eruption maturation of enamel and exposed root surfaces is most important for susceptible mineral phases to be modified by incorporation of soluble fluoride from the plaque into dental hydroxyapatite..$^{1-5}$

Modern prospective caries studies require the measurement of small changes in tooth mineral content. Quantitative measurements of changes in mineral content in a single caries lesion are desirable. One of the most recent techniques is Scanning Electron Microscope (SEM) with Energy Dispersive X-ray analysis (EDAX) attachment. It is a micro-analytical technique that is employed to estimate quantitatively the amounts of mineral in a given tooth sample. ${ }^{5}$

Acidulated Phosphate Fluoride gel, Tooth Mousse Plus, and Remin+ have been found to increase the fluoride content in teeth and thus resist enamel demineralization and are most commonly used. Hence, This study was carried out to evaluate the efficacy of three remineralizing fluids i.e., Acidulated Phosphate Fluoride gel, Tooth Mousse Plus, and Remin+ in resisting demineralization of tooth enamel.

\section{METHODS}

Forty caries free freshly extracted Human maxillary incisors were obtained and stored in normal saline until further use. $3 \mathrm{~mm} \times 3 \mathrm{~mm}$ wax pieces were cut from a modeling wax sheet and placed in the middle third of the labial surface of all the forty incisors to form a window. Except on the wax sheet all around the crown surface acid resistant nail varnish was coated in 2 layers. After the nail varnish dried, red colored nail paint was used as a third coat to differentiate the central window from adjacent area. Once the paint dried the wax sheet was removed and the samples were ready with a exposed, central isolated window of enamel.
The forty incisors are divided in to 4 groups of 10 samples each.

Group-I - samples which were not treated with any remineralizing fluid and taken as control.

Group-II - samples treated with Acidulated Phosphate Fluoride gel (Fluorovil, VISHAL, INDIA) for two minutes.

Group-III - samples treated with Tooth Mousse Plus (GC Corp) for two minutes.

Group-IV - samples treated with Remin+ (Dentsply, Raintree Essex, California) for two minutes.

All the samples in 4 groups were kept separately in artificial saliva (Glandsone, Synthetic Saliva, U.K) for 24 hours and then subjected to demineralization by keeping in Modified Ten Cate solution for 10 days. The samples were sectioned in longitudinal and transverse planes passing through the window of enamel using a hard tissue microtome (LEICA SP 1600). The prepared specimens were gold sputtered and viewed under Scanning Electron Microscope (HITACHI) at a magnification of 1500 X. Energy Dispersive X-Ray Analysis (EDAX) was done to estimate the mineral content in the samples. SEM does the structural analysis and elemental analysis is done by energy dispersive $\mathrm{X}$-rays analysis.

Energy Dispersive X-rays Analysis is a micro-analytical technique that is used in conjunction with SEM to estimate quantitatively the amounts of mineral in a given tooth sample. The EDAX $x$-ray detector measures the number of emitted $x$-rays versus their energy. The energy of the $x$-ray is characteristic of the element from which the $x$-ray was emitted. A spectrum of the energy versus relative counts of the detected $\mathrm{x}$-rays is obtained and evaluated for qualitative and quantitative determinations of the elements present in the specimen using a computer based programme. ${ }^{5}$

\section{RESULTS}

The SEM images (Fig 1, 2, 3, 4) revealed decrease in pore volume of enamel structure in all the three study groups when compared to the control group. To further evaluate the differences between the study groups, the EDAX analysis (energy dispersive $\mathrm{X}$-rays analysis) was done in which the mineral content (Graph 1, 2, 3, 4) (like calcium, phosphorus, fluoride, magnesium and sodium) in weight $\%$ of the tooth samples were quantified.

The EDAX analysis results were subjected to ANOVA test which showed statistically significant ( $p$ value $<0.005$ ) increase in fluoride content in all the treatment groups compared to the control group (Table 1) and statistically insignificant ( $p$ value $>0.005$ ) change in calcium content (Table 2). Among the treatment groups there is statistically significant increase in fluoride content between Group II and III, and Group II and Group IV. But there is no statistically significant difference in fluoride content between Group III and IV. The three groups of remineralizing agents, Acidulated Phosphate Fluoride gel, Tooth Mousse Plus and 


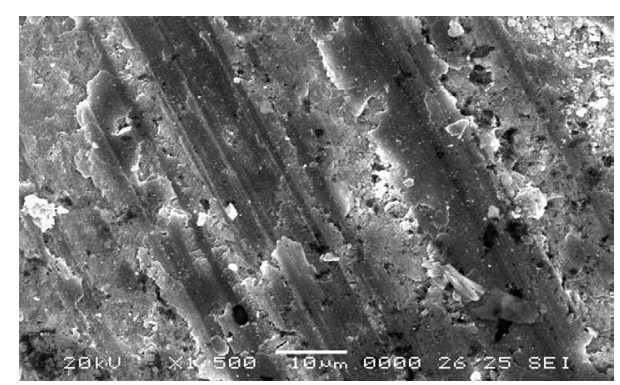

Figure 1. Scanning electron microscopic images - group I.

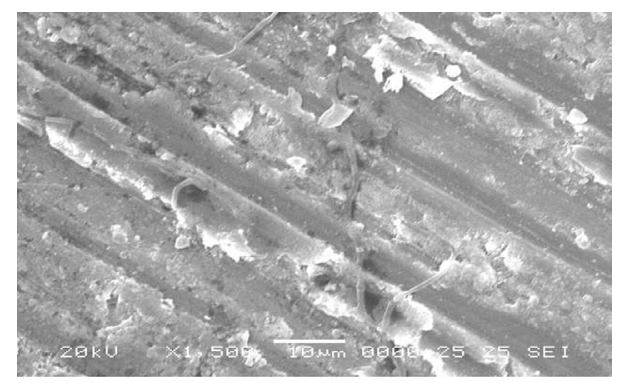

Figure 3. Scanning electron microscopic images - group III.

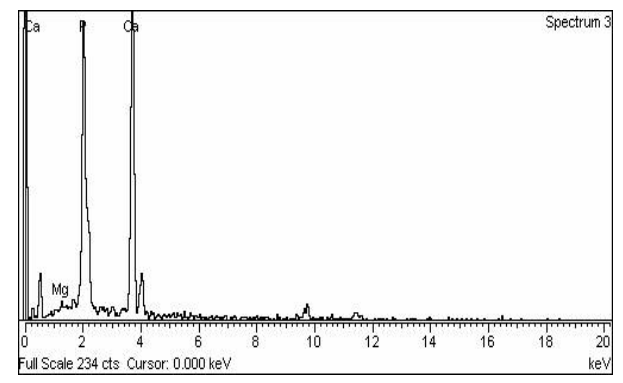

Graph 1. Graph showing SEM -- EDAX values - group I.

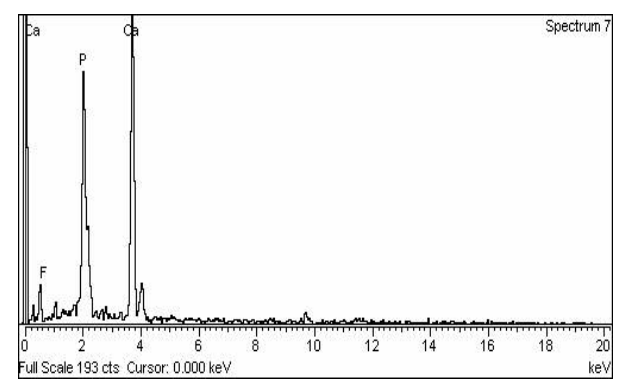

Graph 3. Graph showing SEM -- EDAX values - group III.

Table 1. Table showing Tukey hsd\% test for fluoride wt\%.

\begin{tabular}{c|cc}
\hline GROUP 1 & GROUP 2 & 0.121 \\
& GROUP 3 & $<0.001$ \\
\hline GROUP 2 & GROUP 4 & $<0.001$ \\
\hline \multirow{2}{*}{ GROUP 3 } & GROUP 3 & $<0.001$ \\
\hline & GROUP 4 & 0.005 \\
\hline
\end{tabular}

Remin + showed significant increase in fluoride content and negligible increase in calcium content.

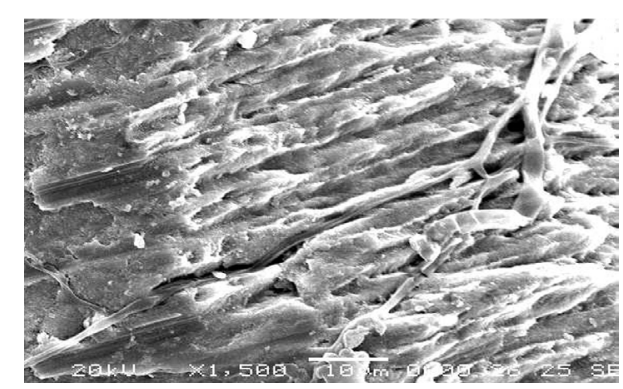

Figure 2. Scanning electron microscopic images - group II.

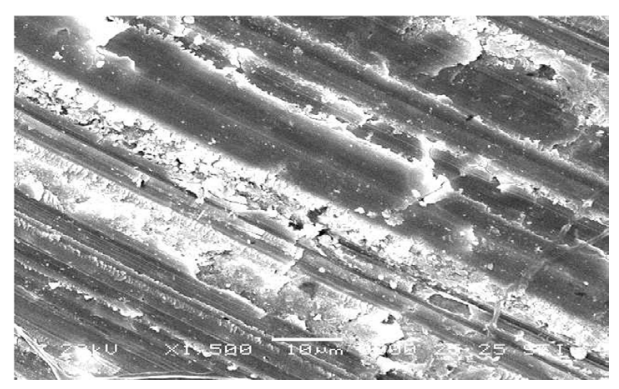

Figure 4. Scanning electron microscopic images - group IV.

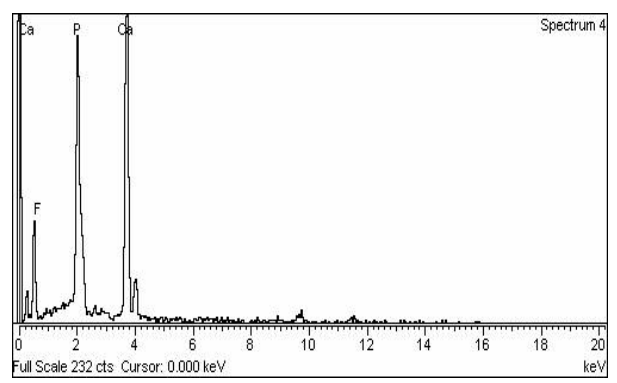

Graph 2. Graph showing SEM -- EDAX values - group II.

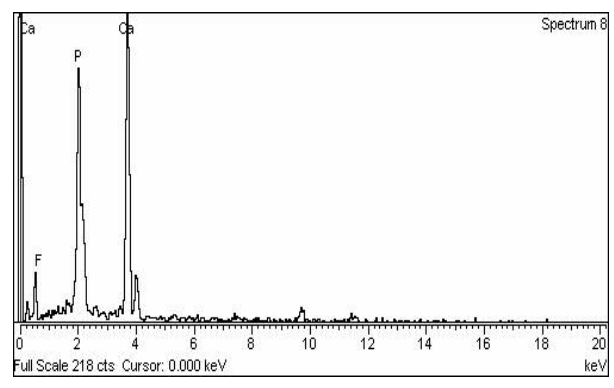

Graph 4. Graph showing SEM -- EDAX values - group IV.

Table 2. Table showing Tukey hsd\% test for calcium wt\%.

\begin{tabular}{|l|ll}
\hline GROUP 1 & GROUP 2 & 0.712 \\
& GROUP 3 & 0.013 \\
\hline GROUP 2 & GROUP 4 & 0.299 \\
& GROUP 3 & 0.149 \\
\hline GROUP 3 & GROUP 4 & 0.890 \\
\hline
\end{tabular}




\section{DISCUSSION}

For many years caries was thought to consist of a one-way progressive demineralization of enamel crystallites followed by degradation of dentin, leading to cavity formation. Dental hard tissues are constantly undergoing cycles of demineralization (when $\mathrm{pH}$ is low) and remineralization (when conditions favor) leading to variations in mineral status of the teeth throughout the day. ${ }^{1}$

In the present study incisors were selected because of wide surface area of enamel on labial surface of the teeth. The mid coronal region was chosen for the windows in order to avoid both the enhanced fluoride surfaces of cervical enamel and the reduced fluoride surfaces coronally, the latter being due to the loss of original surface through wear. $^{6-16}$

Acid resistant nail varnish was used very frequently in the previous studies to cover the enamel surface leaving a window of enamel. ${ }^{10,16-18}$ Nail polish - red color was used to differentiate the enamel window from rest of the enamel which is covered by acid resistant nail varnish (colorless). Synthetic saliva was used to stimulate the oral cavity environment. ${ }^{18}$ Modified Ten Cate's solution was prepared in biochemistry laboratory according to the composition specified by John Hicks with a composition of $2.2 \mathrm{mmol}$ calcium, $2.2 \mathrm{mmol}$ phosphate, $5.0 \mathrm{mmol}$ fluoride, $\mathrm{pH} 3.75$ and used to demineralize the enamel structure. ${ }^{1}$ APF was first introduced by Brudevold at Forsyth Dental Care in the 1960s. ${ }^{1}$ Currently available APF preparation contain sodium fluoride [NaF] as the active ingredient. The general formulation has $1.23 \%$ of fluoride as sodium fluoride [NaF], buffered to a $\mathrm{pH}$ of 3-4 in a 0.1M phosphoric acid. ${ }^{7,19}$

The presence of low-level fluoride influences the transformation of less stable, more soluble mineral phases (dicalcium phosphate dihydrate [DCPD], octacalcium phosphate [OCP], tricalcium phosphate [TCP]) to more stable, less soluble mineral phases (hydroxyapatite [HAP], fluorhydroxyapatite [FHAP], fluorapatite [FAP]). When high levels of fluoride are available, calcium fluoride will be created, and if acid phosphate or phosphate ions are present, calcium fluoride can hydrolyze to form partially fluoridated hydroxyapatite (FHAP).

A substantial volume of literature now exists, demonstrating an anticariogenic effect of dairy products like milk concentrates and cheeses. The casein phosphopeptides - amorphous calcium phosphate nano complexes (CPP$A C P$ ) have been shown to localize at the tooth surface and prevent enamel demineralization in laboratory, animal and human in-situ trials. The CPP-ACP has also been shown to remineralize enamel subsurface lesions in-situ when delivered in oral care products. The proposed anticariogenic mechanism for CPP-ACP is the localization of amorphous calcium phosphate (ACP) at the tooth surface which buffers the free calcium and phosphate ion activities, thereby helping to maintain a state of super saturation with respect to tooth enamel, enhancing remineralization and preventing demineralization. The casein phosphopeptide-amorphous calcium phosphate interacts with fluoride ions to produce an amorphous calcium fluoride phosphate stabilized by the CPP in the tooth surface. This provides soluble calcium, fluoride and phosphate ions to promote remineralization with fluorapatite that is more resistant to future acid challenge. ${ }^{5}$ This could be the probable reason for the group III samples to resist the demineralization of enamel in an acidic solution. The addition of casein phospho pepetide amorphous calcium phosphate nanocomplexes (Recaldent, CASRn691364-49-5) to chewing gum has been suggested to have an additive effect on the remineralization of enamel substrate lesions to that of salivary stimulation. ${ }^{20-22}$

The Remin+ provided additional protection against caries formation in sound enamel compared with topical fluoride treatment. The Remin+ provided calcium, phosphate and fluoride ions for incorporation into clinically and macroscopically undetectable demineralized and hypomineralized enamel prior to lesion formation.

With a relatively low fluoride level and the presence of calcium and phosphate ions, it would be expected that fluoride would enhance transformation of more soluble, less stable mineral phases (DCPD, OCP, TCP) to less soluble, more stable mineral phases (HAP, FHAP, FAP). The additional exposure to synthetic saliva containing calcium and phosphate ions may have allowed for prolonged transformation to more stable, less soluble mineral phases. It is possible that a certain amount of calcium fluoride was formed. With the availability of calcium and phosphate ions from synthetic saliva, calcium fluoride may have hydrolyzed to FHAP. In our present study, in group IV the formation of FHAP could be the most probable reason for it to be resistant against demineralization in acidic environment.

It has been shown that fluoride levels as low as $0.03 \mathrm{ppm}$ in mineralizing fluids allows remineralization to occur. ${ }^{1}$ For optimal remineralization to occur, a fluoride concentration of $0.08 \mathrm{ppm}$ is required. ${ }^{1}$ If dental plaque or salivary fluoride concentrations can be maintained within this range $(0.03$ to $0.08 \mathrm{ppm}$ ), then remineralization would be favored over demineralization, even in acidic conditions. Interestingly, fluoride in saliva can be retained at concentrations between 0.03 and $0.1 \mathrm{ppm}$ for 2 to 6 hours following use of fluoridated dentifrices, low-dose fluoride mouth rinses, and topical home-use fluoride agents. ${ }^{7}$

In the present study, exposure of sound enamel to the $1.23 \%$ APF gel, tooth mousse plus, and Remin+ prior to lesion formation significantly reduced demineralization compared with the matched controls. The control group, without the advantage of fluoride, received less benefit from the synthetic saliva rinsing. Dental plaque, with its gelatinous nature, tends to retain fluoride for much longer periods than saliva, which is constantly secreted and cleared from the oral cavity. It is likely that the APF gel, tooth mousse plus and Remint, used within the current in vitro study, could provide a substantial and prolonged 
increase in fluoride levels within saliva and dental plaque. This would further facilitate remineralization of existing clinically undetectable demineralized and hypomineralized enamel, as well as white spot lesions. With the $1.23 \%$ APF gel, only periodic professional application at the dental office would be possible. Because the tooth mousse plus and Remin+ are applied daily by the patient, there would be a substantial time period for remineralization to occur on a daily basis. In addition, exogenous calcium and phosphate ions from the tooth mousse plus and Remin + are available for uptake by dental plaque along with the fluoride ions. This could provide for prolonged remineralization of white spot lesions and clinically undetectable demineralized and hypomineralized enamel. The EDAX analysis revealed that there is statistically significant increase in fluoride content in three study groups when compared to the control group. Among the study groups, there was statistically significant difference between group 2 and 3 and group 2 and 4 . But there was no statistically significant difference between group 3 and 4 . This attributes to the conclusion that use of topical fluoride results in highly resistant enamel to demineralization thus preventing future caries. Among the study groups, both Tooth Mousse Plus and Remin+ show

\section{REFERENCES}

1. John Hicks, Catherine Flaitz. Role of remineralizing fluid in in vitro enamel caries formation and progression. Quintessence Int 2007;38:313-319.

2. John Hicks, Franklin Garcia-Godoy \& Catherine Flaitz. Biological factors in dental caries: role of remineralization and fluoride in the dynamic process of demineralization and remineralization (part 3). J Clin Pediatr Dent. 2004;28:203-214.

3. MW. Russell. Potential for vaccines in the prevention of caries lesions. Operative Dentistry. 2001;6:51-60.

4. A. Krobica, W. H. Bowen, S. Pearson, D. A. Young. The effect of cheese snacks on caries in desalivated rats. J Dent Res. 1987;66:1116-1119.

5. Mithra N. Hegde, Shishir Shetty, Deepak Pardal. Remineralization of enamel sub-surface lesion using casein phosphopeptide amorphous calcium phosphate. Journal of Conservative Dentistry. 2007;10:19-25.

6. E. C. Reynolds, A. Del Rio. Effect of casein and whey-protein solutions on caries experience and feeding patterns of the rat. Arch oral Biol. 1984;29:927-933.

7. John Hicks, Catherine M. Flaitz, Leon M. Silverstone. Fluoride uptake in vitro of sound enamel and caries-like lesions of enamel from fluoride solutions of relatively low concentration. J Pedod. 1986;11:47-61.

8. Helena Moller, Ulla Schroder. Early Natural Subsurface Caries. A SEM study of the enamel surface before and after remineralization. Caries Res. 1986;20:97-102.

9. H. Reintsema, A. Loading, J. Arends. Fluoridation of partially demineralized Human enamel in vivo. Caries Res. 1986;20:419-123.

10. D. J White, J. D. B. Featherstone. A longitudinal microhardness analysis of fluoride dentifrice effects on lesion progression in vitro. Caries Res. 1987;21:502-512.

11. Joop Arends, Jan Ruben. Fluoride release from a composite resin. Quintessence Int. 1988;19:513-514.

12. Leon M. Silverstone, M. John Hicks, Mary J. Featherstone. Dynamic factors affecting lesion initiation and progression in human dental enamel. II. Surface morphology of sound enamel and caries like lesions of enamel. Quintessence Int. 1988;19:773-785. greater effect than APF gel on the enamel resistance to demineralization/ future carious lesions.

\section{CONCLUSION}

APF gel, Tooth Mousse Plus and Remin + showed significant increase in fluoride content and negligible increase in calcium content. Fluoride in the enamel probably leads to greater resistance against enamel demineralization. Further in vivo studies are to be under taken to evaluate various remineralizing agents efficiency in increasing resistance of enamel to demineralization. The results would have been more promising; if all the samples were subjected for mineral content evaluation before the remineralizing agents were applied and then compared with the mineral content values in the samples after the application of remineralizing fluids.

\section{ACKNOWLEDGEMENT}

We express our sincere thanks to Dr. Inaamul Haq for helping with statistical analysis and Dr. Vijaya Bhaskar Professor, Department of Biochemistry, Mamata Medical College and Hospital for helping us in preparing Modified Tencate's solution required for the study.

13. Jacob M. ten Cate. Current concepts on the theories of the mechanism of action of fluoride. Acta Odontol Scand. 1999;57:325-329.

14. Featherstone JDB. Prevention and reversal of dental caries: role of low level fluoride. Community Dent Oral Epidemiol. 1999;27:31-40.

15. John Hicks, Franklin Garcia-Godoy, Catherine Flaitz. Biological factors in dental caries enamel structure and the caries process in the dynamic process of demineralization and remineralization (part 2). $J$ Clin Pediatr Dent. 2004;28:119-124.

16. J. E. Tyler, D. F. G. Poole. Uptake of fluoride by Human surface enamel from ammonium bifluoride and consequent reduction in the penetration IN VITRO by caries-like lesions. Arch oral Biol. 1984;29:971-974.

17. R. P. Shellis. Relationship between Human enamel structure and the formation of caries-like lesions IN VITRO. Arch oral Biol. 1984;29:975981.

18. A. S. AL-Heal, S. R. Armstrong, X. J. Xie, J. S. Wefel. Effect of smear layer on root demineralization adjacent to resin-modified glass ionomer. $J$ Dent Res. 2003;82:146-150.

19. Soben Peter. Fluorides in preventive dentistry In: Soben Peter, Essentials of preventive and community dentistry. 2nd edn. New Delhi: Arya;2003. pg 312-313.

20. E. C. Reynolds, C. J. Cain, F. L. Webber, C. L. Black, P. F. Riley, I. H. Johnson et al. Anticariogenicity of calcium phosphate complexes of tryptic casein phosphopeptides in the rats. J Dent Res. 1995;74:12721279.

21. E. C. Reynolds. Remineralization of enamel subsurface lesions by casein phosphopeptide-stabilized calcium phosphate solutions. J Dent Res. 1997;76:1587-1595.

22. P. Shen, F. Cai, A. Nowicki, J. Vincent, E. C. Reynolds. Remineralization of enamel subsurface lesions by sugar-free chewing gum containing casein phosphopeptide-amorphous calcium phosphate. J Dent Res. 2001;80:2066-2070. 